\title{
Digital intravascular pressure wave recording during endovascular treatment reveals abnormal shunting flow in vertebral venous fistula of the vertebral artery: illustrative case
}

\author{
Yoshiteru Shimoda, MD, PhD, ${ }^{1}$ Shinya Sonobe, MD, PhD, ${ }^{1}$ Kuniyasu Niizuma, MD, PhD, ${ }^{1,2,3}$ Toshiki Endo, MD, PhD, ${ }^{1,2}$ \\ Hidenori Endo, MD, PhD, ${ }^{1}$ Mayuko Otomo, MD, ${ }^{1}$ and Teiji Tominaga, MD, PhD ${ }^{1}$
}

Departments of ${ }^{1}$ Neurosurgery and ${ }^{3}$ Neurosurgical Engineering and Translational Neuroscience, Tohoku University Graduate School of Medicine, Sendai, Japan; and ${ }^{2}$ Department of Neurosurgical Engineering and Translational Neuroscience, Graduate School of Biomedical Engineering, Tohoku University, Sendai, Japan

\begin{abstract}
BACKGROUND An arteriovenous fistula is an abnormal arteriovenous shunt between an artery and a vein, which often leads to venous congestion in the central nervous system. The blood flow near the fistula is different from normal artery flow. A novel method to detect the abnormal shunting flow or pressure near the fistula is needed.

OBSERVATIONS A 76-year-old woman presented to the authors' institute with progressive right upper limb weakness. Right vertebral angiography showed a fistula between the right extracranial vertebral artery (VA) and the right vertebral venous plexus at the $\mathrm{C} 7$ level. The patient underwent endovascular treatment for shunt flow reduction. Before the procedure, blood pressures were measured at the proximal VA, distal VA near the fistula, and just at the fistula and drainer using a microcatheter. The blood pressure waveforms were characteristically different in terms of resistance index, half-decay time, and appearance of dicrotic notch. The fistula was embolized with coils and $\mathrm{N}$-butyl cyanoacrylate solution.
\end{abstract}

LESSONS During endovascular treatment, the authors were able to digitally record the vascular pressure waveform from the tip of the microcatheter and succeeded in calculating several parameters that characterize the shunting flow. Furthermore, these parameters could help recognize the abnormal blood flow, allowing a safer endovascular surgery.

https://thejns.org/doi/abs/10.3171/CASE21172

KEYWORDS intravascular pressure wave; dicrotic notch; endovascular surgery; arteriovenous fistula

Intravascular pressure at the tip of the catheter could be easily measured by connecting a pressure transducer device to the catheter. Observing the mean intravascular pressure during endovascular surgery for shunting diseases has been determined to be useful. ${ }^{1-10}$ The mean arterial pressure is found to decrease at the feeder of an arteriovenous malformation (AVM) and to increase as the flow embolization progresses, which indicates the treatment's success. ${ }^{1-4}$

Aside from the absolute mean intraarterial pressure, abnormal shunt flow is changed from normal flow in several ways. ${ }^{11}$ For example, using Doppler ultrasonography, the resistance index (i.e., systolic velocity minus diastolic velocity over systolic velocity) has been known to decrease in abnormally shunting arteries. ${ }^{12-14}$ Resistance of the vessels is sensitive to the change of flow dynamics according to the downstream structure and could be used to understand the angioarchitecture of shunting diseases. ${ }^{14-16}$ Therefore, by precisely recording the pressure waveform from the tip of the microcatheter, it could be possible not only to measure the mean pressure but also to extract useful information by analyzing the waveform itself.

An arteriovenous fistula (AVF) is an abnormal arteriovenous shunt between an artery and a vein, which could often lead to venous congestion in the cerebrum or spinal cord. To treat AVF, transarterial/ transvenous embolization or shunt occlusion is used. In the case of

ABBREVIATIONS AVF = arteriovenous fistula; $A V M=$ arteriovenous malformation; $d A V F=$ dural arteriovenous fistula; $V A=$ vertebral artery INCLUDE WHEN CITING Published July 12, 2021; DOI: 10.3171/CASE21172.

SUBMITTED March 31, 2021. ACCEPTED April 20, 2021.

(C) 2021 The authors, CC BY-NC-ND 4.0 (http://creativecommons.org/licenses/by-nc-nd/4.0/). 
transarterial embolization, a passing artery should be recognized to avoid cerebral infarction. Hence, we need a reliable method to determine whether the artery that is going to be embolized is a feeder.

In this study, we performed digital recording of the intravascular pressure waveform from the tip of the microcatheter in a patient who underwent endovascular surgery for vertebral AVF. Moreover, we compared the feature of the pressure waveform with the downstream anatomical structure.

\section{Illustrative Cases}

A 76-year-old woman presenting with progressive right upper limb palsy for 1 year was admitted to our department. Neurological screening demonstrated muscle weakness at the right upper limb innervated by $\mathrm{C} 6-8$. Cervical magnetic resonance imaging showed dilated veins, with flow voids compressing the cervical spinal cord and nerve roots (Fig. 1A). Magnetic resonance angiography presented a significant dilatation of the vertebral venous plexus, which was occluded at the proximal side (Fig. 1B). Right vertebral angiography showed a fistula between the right vertebral artery (VA) and the venous plexus near the root of $\mathrm{C7}$ (Fig. $1 \mathrm{C}$ ). The right VA distal to the fistula was perfused retrogradely from the left VA, whereas the vertebral fistula was fed proximally and retrogradely from the right VA. The proximal side of the draining vein was then occluded. The increased flow was drained through dilated communicating veins: the right deep cervical vein to the right external jugular vein. Accordingly, venous congestion occurred at the C6-8 level of the spinal cord. We planned the transarterial embolization to reduce the shunt flow of the vertebral AVF by occluding the fistula.

At the beginning of the endovascular surgery, we measured the intravascular pressure at four locations (right proximal VA, right distal VA, arterial side of the fistula, and drainer side of the fistula) using a 1.7-French lumen catheter (Fig. 2). Pressure measurements were referenced to the environmental air pressure at the level of the patient's table. The resistance index, which was calculated by systolic pressure minus diastolic pressure divided by systolic pressure, was determined to be 0.50 at the proximal VA (Fig. 2B), 0.48 at the distal VA (Fig. 2C), 0.12 at the arterial side of the shunting point (Fig. 2D), and 0.11 at the venous side of the shunting point (Fig. 2E). The half-decay time of the maximum to minimum amplitude of the pressure waveform was $225.2 \mathrm{msec}$ at the proximal VA (Fig. 2B), $221.5 \mathrm{msec}$ at the distal VA (Fig. 2C), $338.8 \mathrm{msec}$ at the arterial side of the shunting point (Fig. 2D), and $361.3 \mathrm{msec}$ at the venous side of the shunting point (Fig. 2E). Furthermore, the proximal VA had a clear dicrotic notch in the diastolic phase, whereas the arteries and veins near the shunting fistula had signs of neither the dicrotic notch nor the inflection point of the diastolic slope (Fig. 3).

After decreasing the shunt flow by occluding the right proximal VA using a balloon catheter, we packed from the venous side of the fistula using 12 coils. Moreover, we then locally injected $0.03 \mathrm{ml}$ and $0.06 \mathrm{ml}$ of $30 \%$ and $60 \% \mathrm{~N}$-butyl cyanoacrylate solution, respectively, to decrease the remaining shunt flow. The shunt flow was observed to decrease drastically, and the retrograde flow of the distal right VA became antegrade (Fig. 4).

Although the patient experienced a temporal worsening of $\mathrm{C} 7$ radiculopathy, which may have been due to the mass effect of embolization material, her symptoms of muscle weakness in the right upper limb and C7 radiculopathy gradually improved. She was then discharged 11 days after the surgery.

\section{Discussion}

\section{Observations}

The waveform of the intravascular pressure waves using a microcatheter were different near the shunting artery compared with that of the proximal arterial flow. However, two apparent differences should be considered. The first was the resistance index of the pressure waveform, which was noted to decrease as the recording point was close to the fistula. The resistance index was defined as systolic pressure minus diastolic pressure divided by systolic pressure. If the artery does not have normal capillaries in the downstream region, the resistance will be low. Arteries such as the feeders of AVFs and AVMs are known to have a low resistance index. ${ }^{14,17}$ Although the resistance index could also be calculated from the pressure wave using an intravascular catheter, it has not been reported so far.

The second difference was the dicrotic notch that disappeared in the waveform recorded near the shunting fistula. Because the dicrotic notch is known to be formed from the overlap of forward
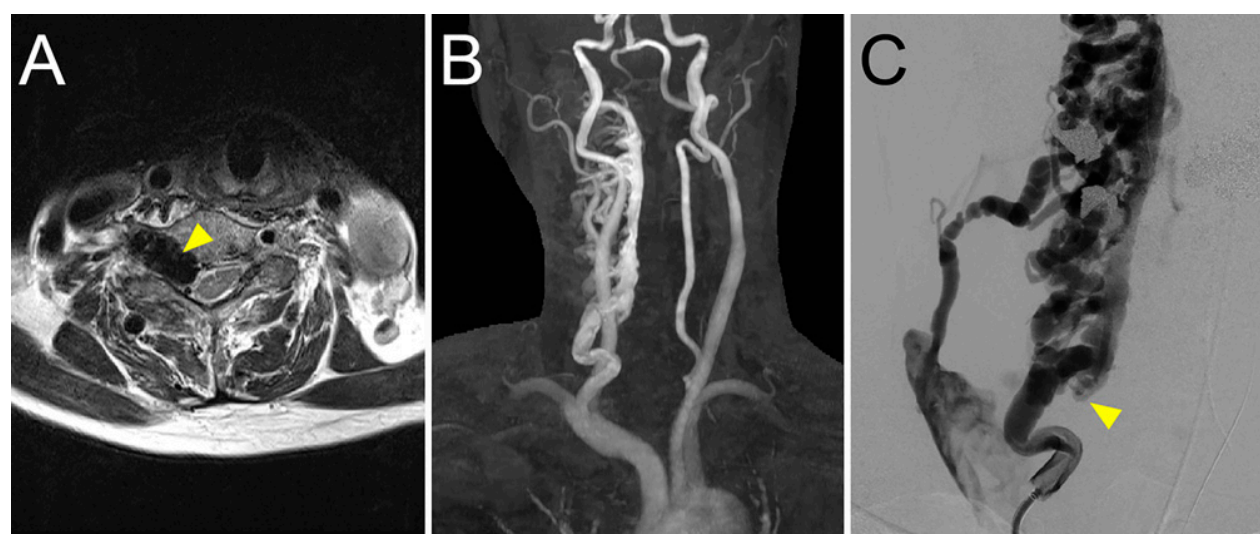

FIG. 1. Preoperative images. A: Preoperative T2-weighted image at the $\mathrm{C} 7$ level. Note that the right vertebral veins are dilated with low-intensity signal (arrowhead). B: Preoperative magnetic resonance angiogram. Note that the right vertebral veins are visible and abnormally dilated. C: Angiogram of the right VA showed the fast appearance of the dilated right vertebral venous plexus. The proximal side of the vertebral vein was occluded (arrowhead). 


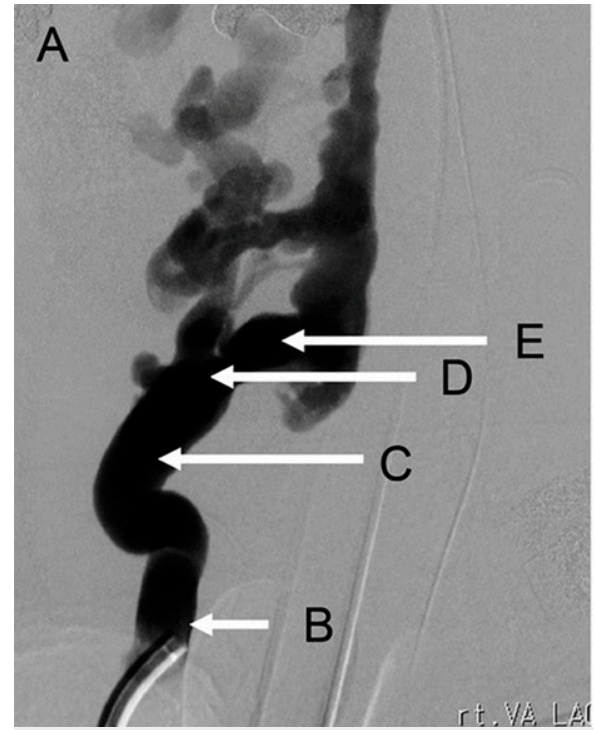

B. Proximal VA

Pressure wave

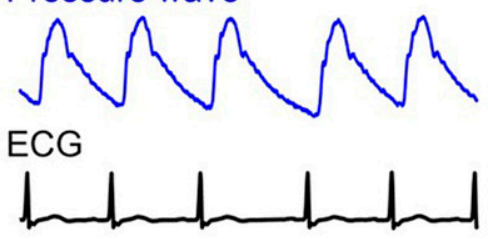

D. Arterial side of the Fistula
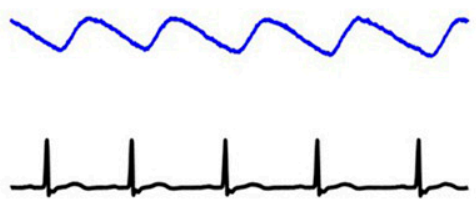

C. Distal VA

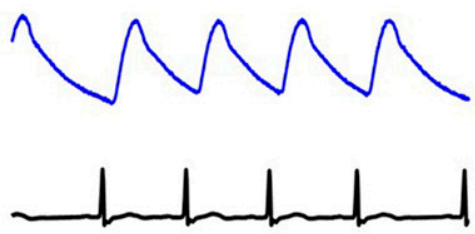

E. Venous side of the Fistula
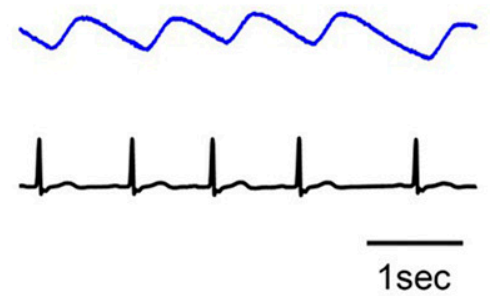

FIG. 2. Pressure wave recording during endovascular surgery. A: Angiogram of the right VA before the endovascular surgery. Intravascular pressures were recorded at each point (B-E). B-E: The pressure wave and electrocardiogram (ECG) were simultaneously recorded for 10-20 sec for each recording point. Note that the waveforms are changing as the recording point gets closer to the fistula. Because all the recordings were performed with the same condition, the amplitude of each pressure wave is comparable.

and backward waves, several factors, such as stroke volumes, peripheral resistance, and aortic distensibility, affect its shape. ${ }^{18-22}$ The dicrotic notch is usually clearly observed at the level of the VA in normal conditions. ${ }^{23}$ Although the dicrotic notch was observed at the origin of the VA in this case, it gradually decreased as the recording point moved toward the shunting fistula and completely disappeared at the shunting point and drainers. This phenomenon could be explained by the fact that resistance downstream of the shunting fistula is decreased compared with that in normal arteries, which have a normal resistance. The decrease of the peripheral

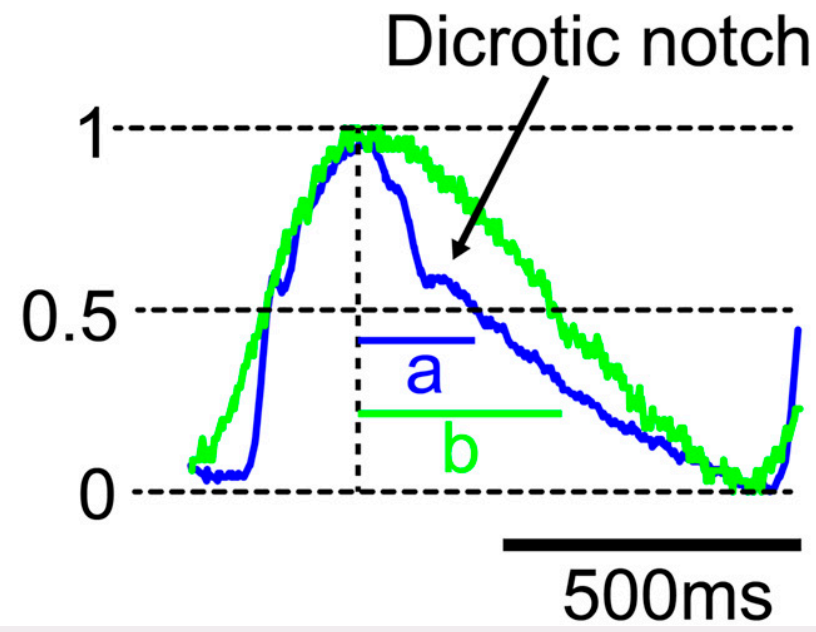

FIG. 3. The waveforms of intravascular pressure that were normalized to the peak pressure at the proximal VA (blue line) and near the fistula (green line) were compared. Note that the wave at the proximal VA has a clear notch, whereas the wave near the fistula does not. The time from the peak amplitude to decay to half amplitude ( $a$ and $b$ ) was prolonged at the fistula. resistance leads to the attenuation of the backward pressure wave and disappearance of the dicrotic notch. Because the existence of the dicrotic wave will alter the pressure slope of the diastolic phase, the half-decay time of the pressure wave will reflect the downstream resistance. We then measured the time from the peak of pressure to halfway $(225.2 \mathrm{msec}$ at the proximal VA vs $338.8 \mathrm{msec}$ at the arterial side of the fistula). On the basis of the form of the normalized waveform (Fig. 3), from the maximal pressure, it took more time to decline to half pressure near the shunting fistula than in the VA proximal to the shunting fistula.

These two aspects of the pressure waveform could also be similarly found in the waveform of flow velocity using Doppler ultrasound with high-flow feeders of AVMs as in past studies. Dempsey et al. measured the flow of AVMs during surgery and compared the parameters from the pre- and postsurgery waveforms. ${ }^{14}$ Although it was not mentioned in their study, the obtained figure clearly showed that the flow speed measurement at the feeder of the AVM artery had an unclear dicrotic notch and increased half-decay time. Thus, the disappearance of the dicrotic notch and increase in half-decay time could be a characteristic marker for shunting flow.

Although we found that the pressure waveforms were quite different according to the recording points, it must be considered that the waveform could be largely affected by the recording settings. ${ }^{24,25}$ First, using a thin catheter will decrease the change between systolic and diastolic pressure. Second, a long catheter will also dampen the pressure pulsation. Moreover, air bubbles and coagulation inside the catheter will change the resistance and the amplitude of the wave. For these reasons, absolute values of systolic and diastolic intravascular pressures are modified strongly by recording conditions. Thus, recording a high-frequency activity, such as a dicrotic notch, should be done with a relatively large and short catheter because it could diminish artificially otherwise. Interpreting the difference between several waveforms correctly is only 


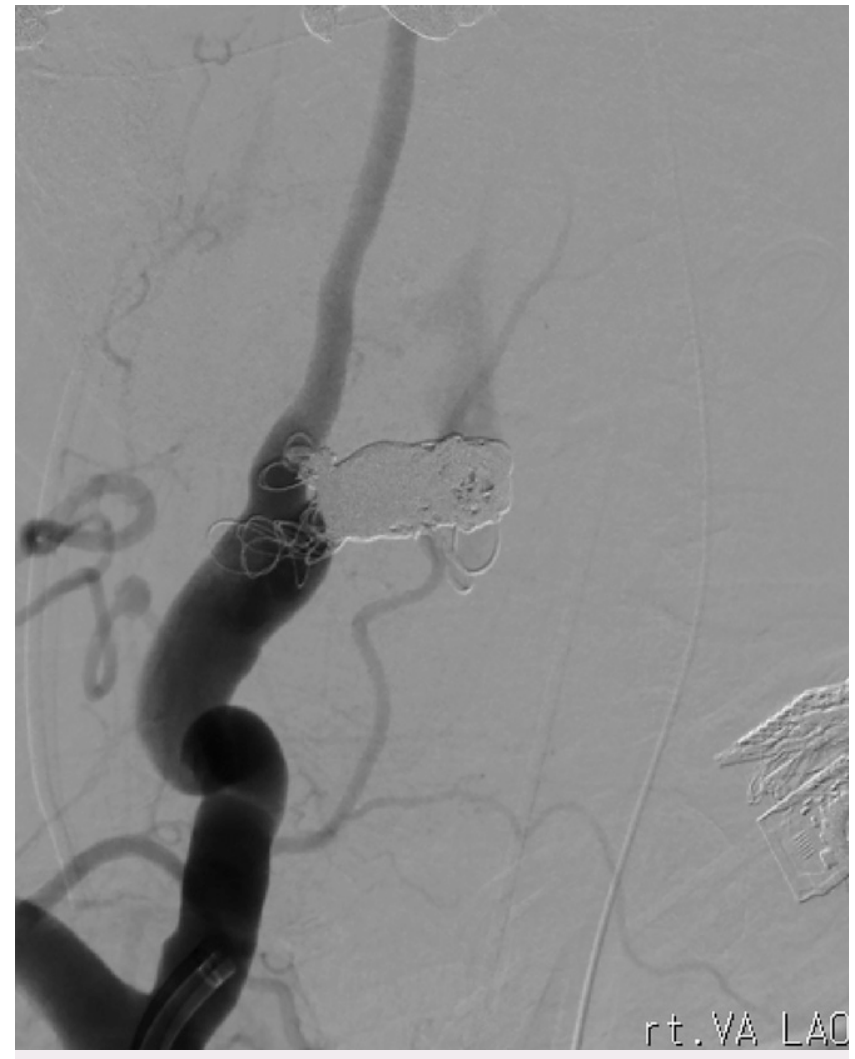

FIG. 4. Postoperative angiogram of the right VA. The flow at the distal VA was normalized, and shunt flow was reduced drastically by the embolized coil.

successful when all the recording conditions are the same between each recording.

The difference in pressure waveform between an arterial shunt and normal vessels could be a strong tool to detect the shunting point in AVMs or dural AVFs (dAVFs). It is sometimes difficult to identify an angiographic shunting point in AVMs or dAVFs with complex angioarchitecture. In addition to the visual information of angiography, quantitative information provided by acquiring pressure waveforms would help neuroendovascular surgeons to decide on a treatment strategy more systematically. Furthermore, these parameters might be useful to validate the effect of endovascular treatment by comparing the pre- and postsurgery values. Although we could not obtain data after the treatment in this case, further data collection and analysis are needed to know the relationship between the parameters and therapeutic efficacy.

\section{Lessons}

The abnormal shunt flow was distinguished from normal flow by measuring the vascular pressure wave with a microcatheter in the case of vertebral venous fistula. This knowledge could be used to know the anatomical structure and differentiate abnormal shunting arteries from normal arteries during endovascular surgery.

\section{Acknowledgments}

This work was supported by Japan Society for the Promotion of Science KAKENHI grant 19K18376.

\section{References}

1. Jungreis CA, Horton JA, Hecht ST. Blood pressure changes in feeders to cerebral arteriovenous malformations during therapeutic embolization. AJNR Am J Neuroradiol. 1989;10(3):575-577.

2. Jungreis CA, Horton JA. Pressure changes in the arterial feeder to a cerebral AVM as a guide to monitoring therapeutic embolization. AJNR Am J Neuroradiol. 1989;10(5):1057-1060.

3. Handa T, Negoro M, Miyachi S, Sugita K. Evaluation of pressure changes in feeding arteries during embolization of intracerebral arteriovenous malformations. J Neurosurg. 1993;79(3):383-389.

4. Henkes H, Gotwald TF, Brew S, et al. Pressure measurements in arterial feeders of brain arteriovenous malformations before and after endovascular embolization. Neuroradiology. 2004;46(8):673-677.

5. Norbash AM, Marks MP, Lane B. Correlation of pressure measurements with angiographic characteristics predisposing to hemorrhage and steal in cerebral arteriovenous malformations. AJNR Am J Neuroradiol. 1994;15(5):809-813.

6. Fogarty-Mack P, Pile-Spellman J, Hacein-Bey L, et al. The effect of arteriovenous malformations on the distribution of intracerebral arterial pressures. AJNR Am J Neuroradiol. 1996;17(8):1443-1449.

7. Miyasaka $Y$, Kurata A, Irikura $K$, et al. The influence of vascular pressure and angiographic characteristics on haemorrhage from arteriovenous malformations. Acta Neurochir (Wien). 2000;142(1):39-43.

8. Miyasaka $Y$, Kurata A, Tokiwa K, et al. Draining vein pressure increases and hemorrhage in patients with arteriovenous malformation. Stroke. 1994;25(2):504-507.

9. Henkes H, Gotwald TF, Brew S, et al. Intravascular pressure measurements in feeding pedicles of brain arteriovenous malformations. Neuroradiology. 2006;48(3):182-189.

10. Joshi KC, Singh D, Tandon MS. Intrafistula pressure measurement in traumatic carotid cavernous fistulas - key to increasing safety and effectiveness of endovascular coiling. Acta Neurochir (Wien). 2014;156(9):1695-1700.

11. Chan KH, Dearden NM, Miller JD, et al. Transcranial Doppler waveform differences in hyperemic and nonhyperemic patients after severe head injury. Surg Neurol. 1992;38(6):433-436.

12. Petty GW, Massaro AR, Tatemichi TK, et al. Transcranial Doppler ultrasonographic changes after treatment for arteriovenous malformations. Stroke. 1990;21(2):260-266.

13. Burkhardt T, Siasios G, Schmidt NO, et al. Intraoperative microDoppler in cerebral arteriovenous malformations. J Neurol Surg $A$ Cent Eur Neurosurg. 2015;76(6):451-455.

14. Dempsey RJ, Moftakhar R, Pozniak M. Intraoperative Doppler to measure cerebrovascular resistance as a guide to complete resection of arteriovenous malformations. Neurosurgery. 2004;55(1):155-161.

15. Shakur SF, Amin-Hanjani S, Mostafa H, et al. Relationship of pulsatility and resistance indices to cerebral arteriovenous malformation angioarchitectural features and hemorrhage. J Clin Neurosci. 2016;33:119-123.

16. Shakur SF, Amin-Hanjani S, Abouelleil M, et al. Changes in pulsatility and resistance indices of cerebral arteriovenous malformation feeder arteries after embolization and surgery. Neurol Res. 2017;39(1):7-12.

17. Spetzler RF, Hargraves RW, McCormick PW, et al. Relationship of perfusion pressure and size to risk of hemorrhage from arteriovenous malformations. J Neurosurg. 1992;76(6):918-923.

18. Merillon JP, Lebras Y, Chastre J, et al. Forward and backward waves in the arterial system, their relationship to pressure waves form. Eur Heart J. 1983;4(suppl G):13-20.

19. Westerhof N, Sipkema P, van den Bos GC, Elzinga G. Forward and backward waves in the arterial system. Cardiovasc Res. 1972;6(6): 648-656.

20. Weber BB, Kerr ME, Wechsler LR, Marion DW. Absence of a diastolic velocity notch does not indicate hyperemia in traumatic brain injured patients without elevated cerebral blood flow velocity. J Neurosurg Anesthesiol. 2002;14(4):279-286. 
21. Lewis $T$. The factors influencing the prominence of the dicrotic wave. J Physiol. 1906;34(6):414-429.

22. Murray WB, Foster PA. The peripheral pulse wave: information overlooked. J Clin Monit. 1996;12(5):365-377.

23. Zhang $\mathrm{C}$, Chau N, Ho H. Patient-specific blood flow analysis for cerebral arteriovenous malformation based on digital subtraction angiography images. Front Bioeng Biotechnol. 2020;8:775.

24. Henkes H, Felber SR, Wentz KU, et al. Accuracy of intravascular microcatheter pressure measurements: an experimental study. $\mathrm{Br} \mathrm{J}$ Radiol. 1999;72(857):448-451.

25. Shapiro GG, Krovetz LJ. Damped and undamped frequency responses of underdamped catheter manometer systems. Am Heart J. 1970;80(2):226-236.

\section{Disclosures}

Dr. Tominaga reports grants from Ministry of Education, Culture, Sports, Science and Technology during the conduct of the study; and grants from Ministry of Education, Culture, Sports, Science and Technology and grants from Ministry of Health, Labor and Welfare outside the submitted work.

\section{Author Contributions}

Conception and design: Shimoda, Niizuma. Acquisition of data: Shimoda, Sonobe, Niizuma, Otomo. Analysis and interpretation of data: Shimoda, T Endo, H Endo. Drafting the article: Shimoda. Critically revising the article: Shimoda, Niizuma, T Endo. Reviewed submitted version of manuscript: Shimoda, Niizuma, T Endo, H Endo, Tominaga. Approved the final version of the manuscript on behalf of all authors: Shimoda. Administrative/technical/material support: Shimoda. Study supervision: Tominaga.

\section{Correspondence}

Yoshiteru Shimoda: Tohoku University Graduate School of Medicine, Sendai, Japan. y.shimoda@nsg.med.tohoku.ac.jp. 